\title{
Balance recognition on the basis of EEG measurement
}

\author{
Natalia Tusk \\ Gdańsk University of Technology \\ ul. Narutowicza 11/12 \\ 80-233 Gdańsk, Poland \\ email: tusk.nat@gmail.com
}

\author{
Artur Poliński \\ Gdańsk University of Technology \\ ul. Narutowicza 11/12 \\ 80-233 Gdańsk, Poland \\ email: apoli@biomed.eti.pg.gda.pl
}

\author{
Tomasz Kocejko \\ Gdańsk University of Technology \\ ul. Narutowicza 11/12 \\ 80-233 Gdańsk, Poland \\ email: tomkocej@pg.gda.pl
}

\begin{abstract}
Although electroencephalography (EEG) is not typically used for verifying the sense of balance, it can be used for analysing cortical signals responsible for this phenomenon. Simple balance tasks can be proposed as a good indicator of whether the sense of balance is acting more or less actively. This article presents preliminary results for the potential of using EEG to balance sensing. The results are not unequivocal and further research is required.
\end{abstract}

\section{INTRODUCTION}

$\mathrm{S}$ ENSE of balance is an extremely important ability that allows humans to maintain an upright posture or to remain stable in many advanced motion processes. However, the organs responsible for the sense of balance partially lose their function with age, which is a major problem in the aging population. This problem also concerns younger people with dysfunctions or diseases of organs responsible for equilibrioception [1].

People over 65 years old lose balance significantly more frequently than younger people. In this group, one in every three persons experiences one or more falls due to balance loss each year [1]. This can lead to serious injuries and eventually become a life threat. Even if the consequences are not too severe, living with a poor sense of balance can be a serious inconvenience, causing a fear of falling. Other longterm consequences include weak self-reliance, low social and behavioural activity and more limited mobility. For this reason, there is a raising need for sensor-based multimodal systems that provide care and support for older people [2].

Taking into consideration the fact that nowadays the population is aging, sense of balance examinations may help to diagnose and in consequence treat balance disorders. This could raise the standard of living of older or ill people, and prevent many dangerous accidents from happening.

Physiologically, there are a few organs responsible for balance control. The signals' source for the sense of balance originates from the vestibular system, the eyes and proprioceptors. These signals are analysed by the central nervous system, mainly the cerebellum and the brain stem.

This work has been partially supported by statutory funds of the Faculty of Electronics, Telecommunications and Informatics, Gdańsk University of Technology.
The involvement of the cerebral cortex in balance control has also been proven [3], [4]. For this reason, examining the human brain and its functions is of great importance in relation to balance.

Most basic examinations of human balance are neurological tests such as the finger-to-nose test, the Romberg test, the Unterberger and Fukuda stepping test, the Hautant's test or gait analysis [5]. Performing diagnostic procedures with computer assistance helps to gather and interpret more valuable data such as posturography, craniocorpography or the Kinect supported analysis of physical tests. Taking into account the fact that balance loss causes involuntary eye movements called nystagmus, diagnostic procedures can also include: electronystagmography (ENG), electrooculography (EOG), videonystagmography (VNG), infrared reflection oculography (IROG), and the search coil technique. Because one of the most important parts of the equilibrium organ in the human body is the vestibular apparatus, the last type of methods used are audiometric tests, mainly electrocochleography (ECOG) and Brainstem Auditory Evoked Potentials. The least frequently used method for analysing the human balance is electroencephalography (EEG). This is because it is the most high-level method. The cerebral cortex is responsible for analysing all neural signals, including balance sensing. This is why it becomes more and more popular in terms of balance examination [3]. It requires advanced signal processing to obtain the diagnostic information from the EEG signal. The most common technique of EEG signal analysis is the Fourier transform. Such an approach allows analysing different frequency bands separately.

Calculating the Fourier transform of the signal enables the use of Power Spectral Density (PSD) which reflects the distribution of signal power over frequency [6]. PSD has been used for identifying cortical activity changes on the basis of EEG [3].

The aim of the study was to verify if using EEG was possible to differentiate the one and two feet standing position which required more body balancing than the stable both feet posture. 


\section{METHODS}

\section{A. Design of the test and examination conditions}

The tests consisted of two EEG measurement series for each patient. The first one was conducted for a patient who was standing with both feet on the ground maintaining an upright standing posture. The second one was conducted for a patient standing on one foot and also trying to maintain an upright standing posture.

Each measurement series lasted approximately three minutes - all data were cut to the same length during the analysing process. All other external stimuli, apart from one or two feet standing position and vision were excluded.

\section{B. Examined subjects}

Seven volunteers were recruited for the tests, four men and three women. All of them were young - between 20 and 35 years of age - and their sense of balance was supposedly ordinary. They did not have any documented disorders that might have influenced it. Their anatomy also did not indicate any pathological disorders that might have resulted in postural deviations. In order to compare the results for open and closed eyes, some measurement series were conducted for open ( 3 subjects, 1 man and 2 women, patients number 13 ) and some for closed eyes (4 subjects, 3 men and 1 woman, patients number 4-7).

\section{Measurement system structure}

The BioSemi EEG system was used during the measurements [7]. It is a multi-channel, high resolution biopotential measurement system for research applications. The system consisted of: 16 active electrodes as well as two reference electrodes, headcap, AD-box, which is an analogto-digital converter receiving and amplifying the signals coming from the electrodes, USB receiver, which converts the digitized signal into USB output, PC workstation directly connected to the USB receiver, and dedicated software for EEG data analysis.

The electrodes were located in accordance with the manufacturer's suggestions for 16 active electrodes' application. Their location was compatible with standardized 10-20 system for EEG measurement (Fig. 1).

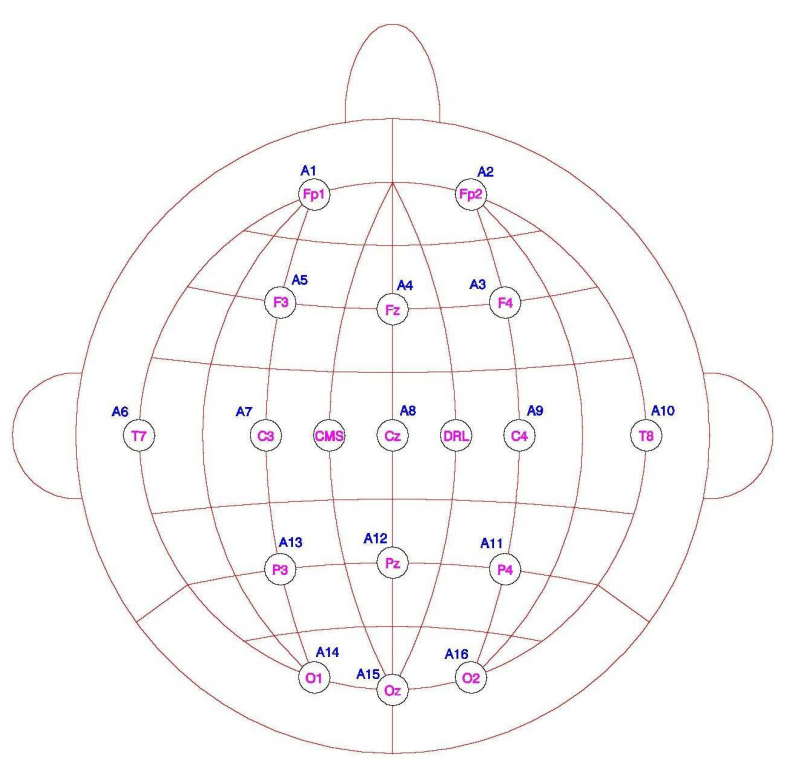

Fig. 1 Electrodes' location during the experiments [7].

The software used for EEG data analysis was ActiView. It is a LabVIEW-based program for the acquisition of EEG signals. The EEG sample rate was set to $2048 \mathrm{~Hz}$. The software also allowed the electrodes' impedance to be checked. It was checked for every subject and more gel was applied in case it was too big.

\section{Data analysis}

All data were processed using the Matlab environment. First, the magnitude squared coherence (MSC) [8] for the two signals (one and two feet standing positions) was calculated using originally and down sampled data

$$
M S C=\left|C_{x y}(\omega)\right|^{2}=\frac{\left|G_{x y}(\omega)\right|^{2}}{G_{x x}(\omega) G_{y y}(\omega)}
$$

where $G_{x x}(\omega), G_{y y}(\omega)$ are the power spectra of these signals, and $G_{x y}(\omega)$ is the cross-power spectrum. These parameters can be obtained by means of the Fourier transform

$$
\begin{aligned}
& G_{x x}(\omega)=|X(\omega)|^{2} \\
& G_{y y}(\omega)=|Y(\omega)|^{2} \\
& G_{x y}(\omega)=X(\omega) Y^{*}(\omega)
\end{aligned}
$$

where $X(\omega)$ and $Y(\omega)$ are the Fourier transforms of one and two feet standing positions' EEG signals. The magnitude squared coherence was calculated to find the most significant frequency band for further analysis. The band selection was also supported by results presented in [3]. Finally, the beta rhythm defined as $13-19 \mathrm{~Hz}$ and sigma defined as $30-40$ $\mathrm{Hz}$ frequency range were chosen.

Next, the power for the selected bands of the signal was calculated using the Fourier transform. Since we are interested in the reaction to increased balance requirements, time changes of the power were estimated. The reference signal was taken as the average power for the selected bands calculated for the two feet standing position. 
In the case of the one foot standing position, the power was calculated for $60 \mathrm{~s}$ and $1 \mathrm{~s}$ time windows shifted through the whole measurement time.

The measurements were conducted with open and closed eyes since in the second case the balance preservation is more complicated.

The power for the selected frequency band was calculated using PSD

$$
\operatorname{PSD}(X)=|X(\omega)|^{2}
$$

where $X(\omega)$ is the Fourier transform of the input $x(t)$ signal.

\section{RESULTS}

In the following figures the relative power versus time is shown. These were normalized in relation to the mean value of both feet standing power for the selected channel.

The relative power for the beta band of channel number 10 for the one foot standing position of patient number 4 for 60 $\mathrm{s}$ and $1 \mathrm{~s}$ averaging windows is shown in Fig. 2. To better show the difference, the power was normalized to the power of $60 \mathrm{~s}$ window.

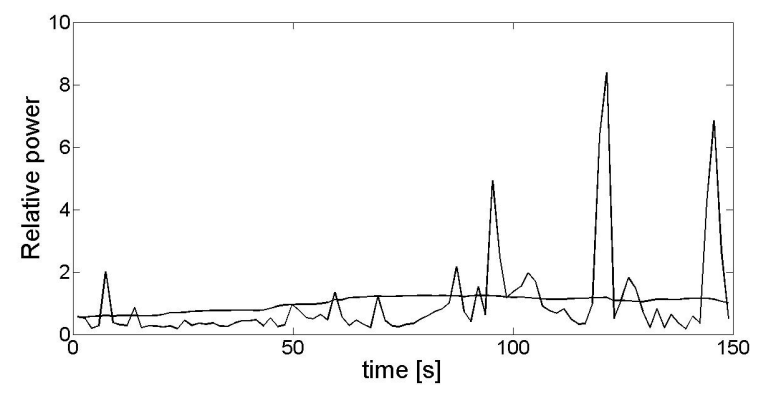

Fig. 2 Example of the length of averaging window influence.

The relative power for the beta band of channel number 10 for the one foot and two feet standing positions of patient number 3 for $60 \mathrm{~s}$ averaging window is shown in Fig. 3.

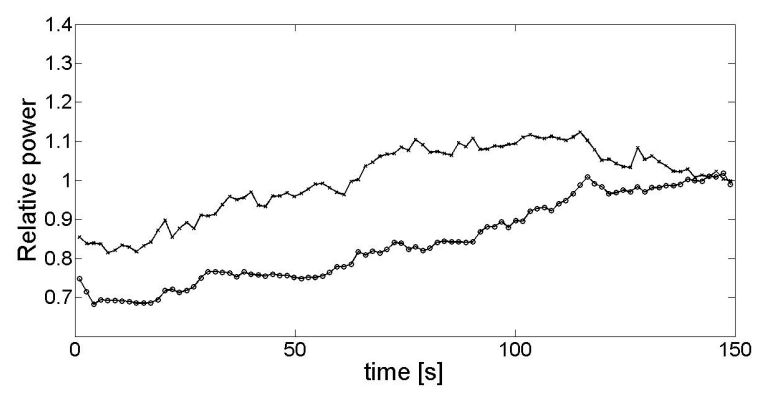

Fig. 3 Example of relative power for the one foot (x) and two feet (o) standing positions for open eyes.

The relative power for the beta band of channel number 10 for the one foot and two feet standing positions of patient number 5 for $60 \mathrm{~s}$ averaging window is shown in Fig. 4.

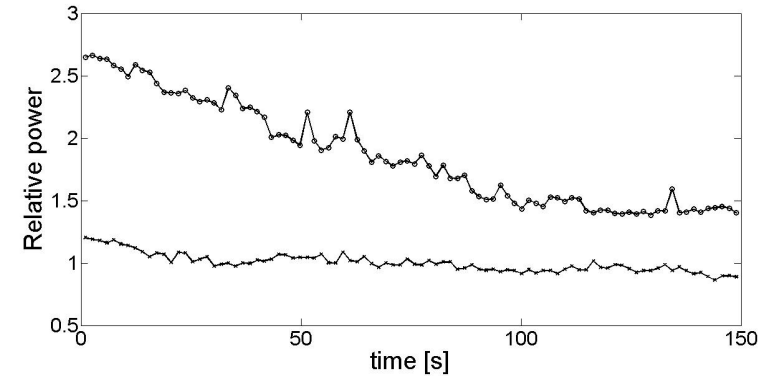

Fig. 4 Example of relative power for the one foot (x) and two feet (o) standing positions for closed eyes.

The relative power for the beta band of channel number 9 for the one foot and two feet standing positions of patient number 7 for $60 \mathrm{~s}$ averaging window is shown in Fig. 5.

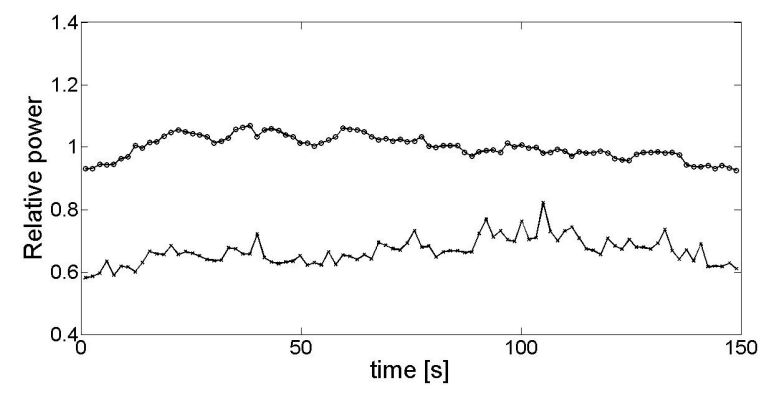

Fig. 5 Example of relative power for the one foot (x) and two feet (o) standing positions for closed eyes.

The relative power for the sigma band of channel number 9 for the one foot and two feet standing positions of patient number 7 for $60 \mathrm{~s}$ averaging window is shown in Fig. 6.

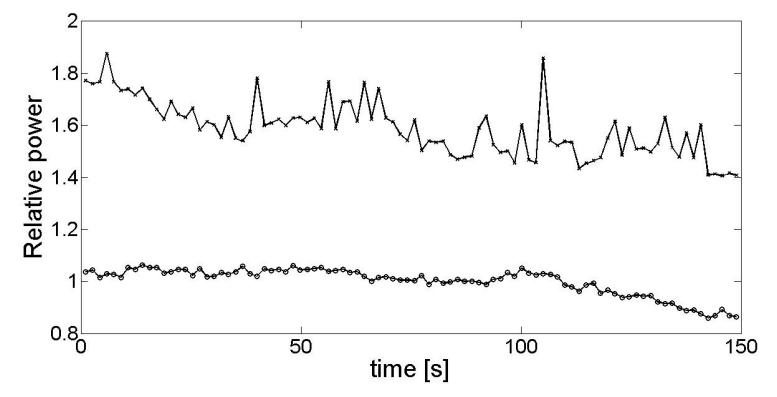

Fig. 6 Example of relative power for the one foot (x) and two feet (o) standing positions for closed eyes.

The relative power for the beta band of channel number 9 for the one foot and two feet standing positions of patient number 4 for $60 \mathrm{~s}$ averaging window is shown in Fig. 7.

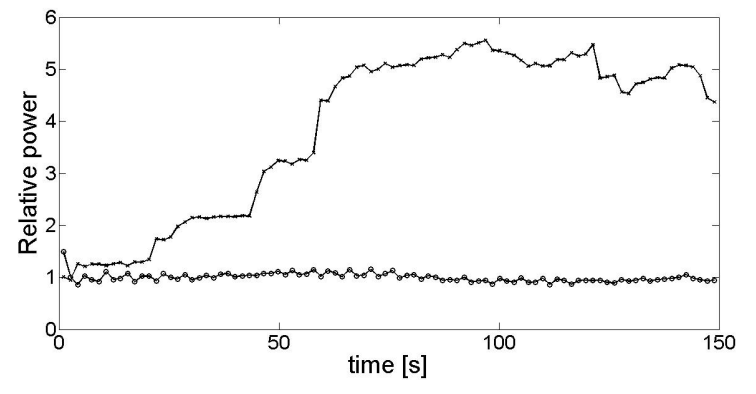

Fig. 7 Example of relative power for the one foot (x) and two feet (o) standing positions for closed eyes. 


\section{DISCUSSION}

The measurement results are not fully consistent. The number of examined subjects might not have been sufficient. Increasing the number of examined subjects may lower the discrepancy between data.

The coherence did not clearly indicate the best frequency band for analysis - neither for any examined electrode, nor for any examined subject. This may be due to complications in its calculations. Because of this, further comparison was conducted for beta and sigma frequency bands according to [3].

Another problem was channel selection. All 16 channels gave different results. Channels 3, 6, 7, 9, 10, and $14-16$ were most meticulously analysed. The results observed are patient dependent, including differences between electrode signals. This might have been caused by physiological differences and a slight difference in the location of electrodes caused by headcap displacements.

The power of the signal can change even for the selected band. One reason is the electrode's placement, while the second one can be due to balance itself. Even in the two feet standing position the signal can vary with time and this variation may be observed in the selected channels. Due to the very tight appliance of the headcap and the corresponding signals in neighbouring electrodes, it is unlikely that the cause are changes in contact impedance.

In the case of the one foot standing position, the signal variation can be observed as well. Some of the variations may be due to the brain's reaction to changes in the patient's position or tiredness caused by the length of the examination.

The problem consists in that in the event of losing balance the lead tension may change, which may cause short time variations in the measurement signal. This should be reduced by averaging power in a 60 -second window (Fig. 2). Such averaging may also prevent the fast signal changes from being observed. This is why the comparison with a 1-second averaging window was carried out. In the case of the 1-second averaging window more rapid changes are visible, however, some of them may be due to distortion such as changes in lead tensions.

The influence of open/closed eyes on the signal registered was also observed. The difference between the one foot and two feet standing position was harder to observe for examinations carried out with eyes open. It was due to the role of the eyes in balance maintenance - it was harder for the patients to remain in an upright posture with the lack of visual stimuli (Figs. 3-4).

The main problem is that measurement data are not consistent. The results depend on the patient, channel and frequency band. However, in most cases there is a difference in signal power for different standing positions. These personal differences in the results obtained may be due to anatomical and physiological differences (especially the sense of balance).

The relation between power for two standing positions for the selected bands is person dependent (Figs. 5-7).

The most consistent results were obtained for closed eyes for channel number 6 and sigma frequency band.

The brain regions responsible for motor functions may also participate in the measured signals. This is why the analysis is so complicated.

According to the results obtained, new tests are prepared based on the evoked potentials (EP) approach. The plan assumes examination using periodic lifting of one foot.

\section{CONCLUSIONS}

The primary results show that it is possible to analyse the sense of balance using EEG, however, the frequency band and channel selection requires more investigation. Careful specification of the measurement protocol to remove unwanted sources of EEG signal also requires further verification - for example of conditions relating to open and closed eyes.

The problem of data analysis may follow from the complicated dependence of EEG signal on many sources like visual, motor aspects, etc.

The time variability of power courses for different time averaging window lengths should be also investigated as a possible indicator of the sense of balance.

It seems that further analysis for a larger number of people would allow to estimate the sense of balance on the basis of single channel or differential EEG measurements using time analysis for selected frequency bands.

\section{REFERENCES}

[1] S. Chaudhuri, H. Thompson, and G. Demiris, "Fall Detection Devices and their Use with Older Adults: A Systematic Review", J Geriatr Phys Ther. 2014; 37(4): 178-196

[2] M. Kaczmarek, A. Bujnowski, J. Wtorek, A. Polinski, "Multimodal Platform for Continuous Monitoring of the Elderly and Disabled", Journal of Medical Imaging and Health Informatics, Volume 2, Number 1, March 2012, pp. 56-63(8)

[3] Y. Yi F. Tse, J. S. Petrofsky, L. Berk, N. Daher, E. Lohman, M. S. Laymon, P. Cavalcanti, "Postural sway and rhythmic electroencephalography analysis of cortical activation during eight balance training tasks", "Med. Sci. Monitor", 2013; 19:175-186

[4] Y. Ouchi, H. Okada, E. Yoshikawa, S. Nobezawa, M. Futatsubashi, Brain activation during maintenance of standing postures in humans. J Neurol, 1999; 122 (Pt 2): 329-38

[5] S. Dejardin, The clinical investigation of static and dynamic balance, BENT, 2008, 4, Suppl. 8, 29-36

[6] A. V. Oppenheim, G. C. Verghese, "Introduction to Communication, Control, and Signal Processing", Ch10, 2010

[7] http://www.biosemi.com/

[8] R. E. Challis, R. I. Kitney, Biomedical signal processing (in four parts). Part 3. The power spectrum and coherence function, Med Biol Eng Comput. 1991 May; 29(3):225-41 